\section{A Versatile and Affordable Plunge Freezing Instrument for Preparing Frozen Hydrated Specimens for Cryo Transmission Electron Microscopy (CryoEM)}

\author{
Linda Melanson \\ Gatan, Inc., Pleasanton, CA \\ Imelanson@gatan.com
}

CryoEM is a powerful tool in the arsenal of structural biologists and soft polymer chemists. Hydrated specimens require a preservation method that will counteract the effects of the electron beam and the high vacuum environment of the electron microscope. Classical specimen preparation techniques using chemical fixatives are not able to capture the native structure of the once hydrated specimen perfectly. In contrast to classical methods for preserving specimens for electron microscopy, rapid freezing of radiation-sensitive specimens such as dispersed biological macromolecular assemblies, 2D crystals, and colloids allows the structural details of the specimen to be captured in their essentially native state to near atomic resolution.

Key to the entire process of cryoEM is the preparation of the specimen. A successful cryo preparation requires that the specimen is rapidly frozen so that all motion and metabolic activities are instantaneously arrested and that the fluid within or surrounding the specimen freezes without the formation of ice crystals.

There are three main steps for preparing samples for cryoEM: - A small aliquot $(3 \mu \mathrm{L})$ of a fluid suspension containing the sample is applied to the surface of a supporting substrate such as a fenestrated carbon film (Quantifoil or C-flat $t^{\mathrm{Tm}}$ ) or a continuous carbon film that is attached to the surface of a standard TEM specimen grid.

- The droplet is blotted with filter paper until only a thin layer (approximately $100 \mathrm{~nm}$ thick) is left on the support substrate.

- The thin fluid layer is rapidly immersed into a suitable cryogen of high heat capacity, which gives instantaneous and contaminant-free freezing.

A suitable cryogen must be capable of achieving a freezing rate of approximately $10^{6}$ Kelvin per second. At room temperature and pressure, the heat capacity of liquid nitrogen (boiling point $-196^{\circ} \mathrm{C}$ ) is too low to properly freeze the specimen; a specimen placed within the liquid nitrogen will cause rapid boiling in the area around the specimen forming an insulating layer of nitrogen gas. The cooling rate of liquid nitrogen can be improved provided that it is maintained at a temperature near its melting point of $-210{ }^{\circ} \mathrm{C}$ by applying a low vacuum for several seconds just prior to freezing the specimen. Alternatively, very rapid cooling for plunge freezing specimens can be obtained using liquid ethane maintained close to its melting point $\left(-181.76^{\circ} \mathrm{C}\right)$ using liquid nitrogen as the primary coolant. $\dagger$

Cryoplunge 3 (also known as Cp3) is Gatan's new, semi-automatic plunge freezing instrument specifically designed for the preparation of frozen hydrated specimens for cryoEM, figure 1 .

Everything is included with the $\mathrm{Cp} 3$ for fast, easy set-up. Weighing only $12.5 \mathrm{~kg}$, the $\mathrm{Cp} 3$ is portable, and its small footprint

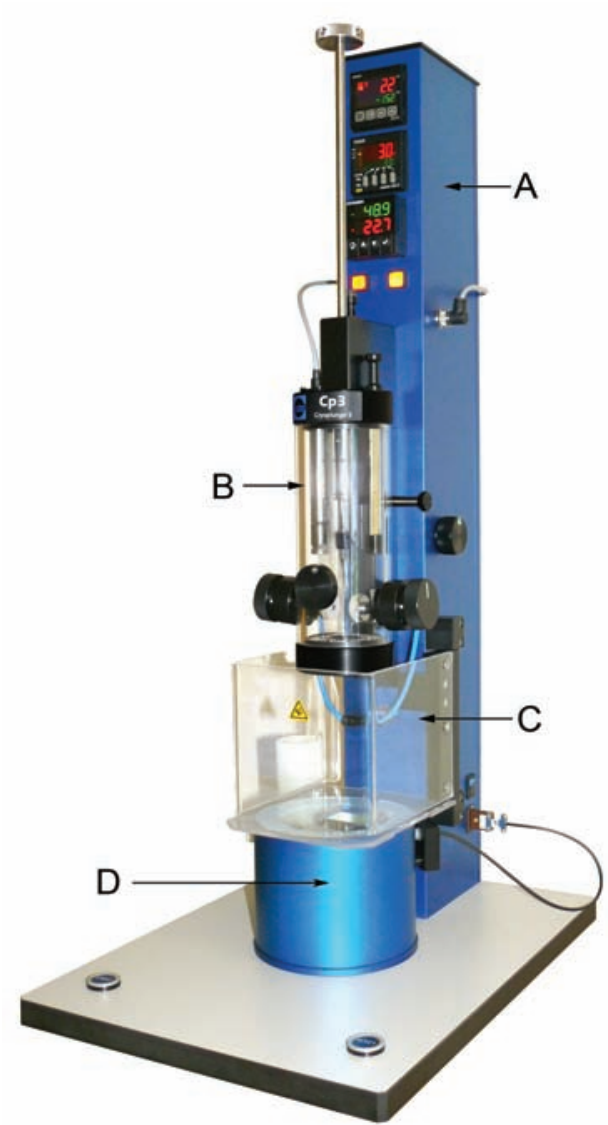

Figure 1: Cryoplunge 3 showing support column (A), humidity chamber (B), safety shield (C), and cryogenic workstation (D).

requires a minimum of space on a standard laboratory bench. The support column of a $\mathrm{Cp} 3$ houses all of the pneumatic logic and electrical components including the ethane temperature controller, the blotting timer and the temperature/humidity meter.

The humidity chamber safeguards the specimen from drying during the blotting process. A simply designed humidity wand

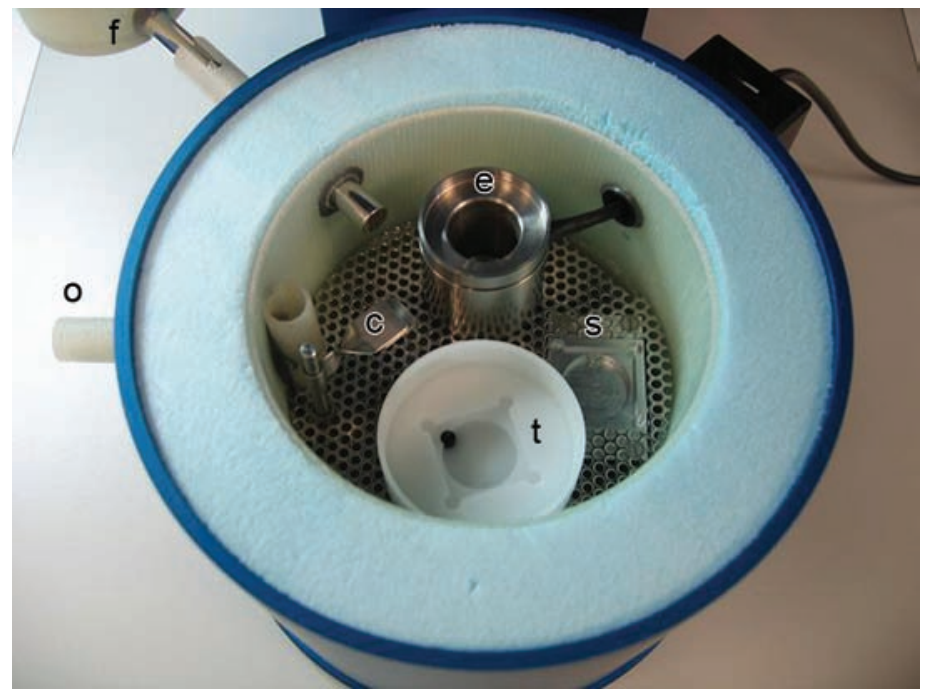

Figure 2: Cryoplunge 3 workstation (shown at room temperature with the workstation covers removed): ethane pot (e), cryo grid box transfer pot ( $t)$ that accepts round or square cryo grid boxes, liquid nitrogen remote fill funnel(f), workstation overflow port (o), cryo grid box staging area (s), filter paper clip (c) for blotting excess ethane from frozen hydrated grid prior to storage or viewing on the TEM. 


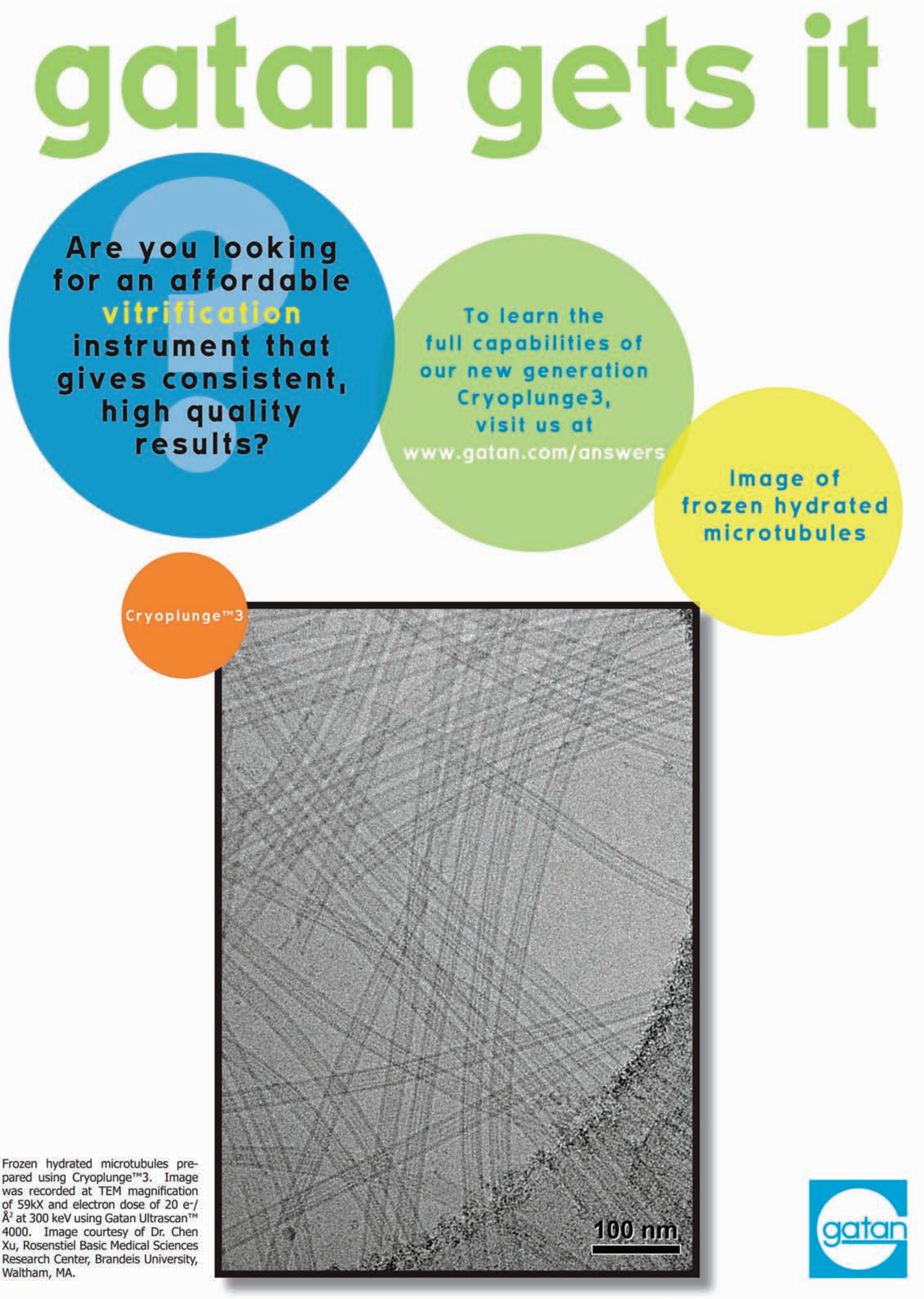



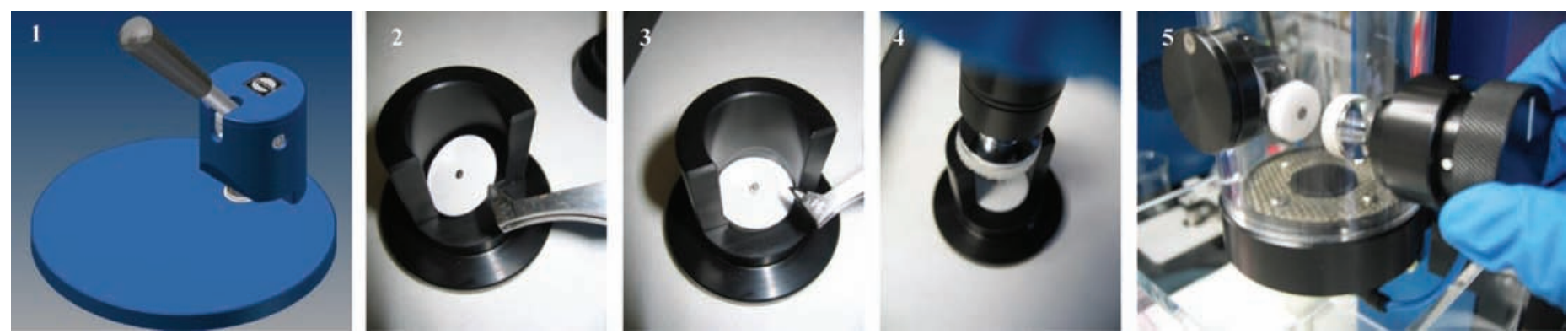

Figure 3: Cp3's filter paper die cutter (1) lets one select the filter paper best suited to the experiment. Mounting the blotting filter paper with the easy-to-use filter paper loading jig: loading the filter paper disk onto the inverted filter paper securing pin (2), loading the protective backer (3), which prevents contamination of the blotter's foam pad during the blot cycle, attaching the filter paper and protective backer onto a blot assembly (4), and inserting the blotter into the humidity chamber (5).

with a replaceable cellulose sponge provides high humidity to the chamber approximately 15 minutes after insertion. A humidity/ temperature sensor monitors the atmospheric conditions within the chamber. The blot assemblies, humidity wand, and the sensor are incorporated into the humidity chamber and all of these components, including the humidity chamber itself, can be easily removed for cleaning.

There are two safety interlocks on Cp3. The safety interlock on the safety shield protects the user during the blotting and plunging cycle. The safety interlock on the cryogenic workstation ensures that the workstation is perfectly aligned with the plunge piston, which will not release unless this safety connection is made.

The specially designed, removable cryogenic workstation, figure 2, maintains the cryogen (ethane) at a temperature just above its melting point to ensure consistent freezing of the specimen. As liquid nitrogen is added to the workstation through the attached funnel, a blanket of cold, dry nitrogen gas fills the workstation chamber. This minimizes condensation of atmospheric oxygen and water vapor onto the surface of the ethane; it also generates a protective cryo interface for transferring the frozen hydrated grid within the workstation.

The adjustable pneumatic blotting action for $\mathrm{Cp} 3$ is triggered by an electronic timer that is accurate to 0.1 seconds. The specimen grid can be blotted one time or multiple times. $\mathrm{Cp} 3$ comes with two interchangeable blot assemblies for two sided blotting and one blanking plug that provides the capability for one-sided front or back side blotting of the specimen grid. By setting the blot timer to zero, one can override the automatic blotting action altogether to facilitate other methods for applying the specimen, which may not require blotting.

The main specimen loading port is in the front of the humidity chamber but the specimen can also be applied to the TEM grid from either the left- or right-hand side of the chamber by removing one of the blotters. A specially designed die cutter allows one to cut the filter paper of their choice for blotting the specimen, Figure 3. The easy to clean filter paper loading jig ensures a virtually contamination free means for loading the blotting filter paper disks onto the blotters.

The pneumatically assisted, multi-positional plunge piston fires the freshly blotted grid into a temperature monitored ethane vessel at $1.7 \mathrm{~m} / \mathrm{sec}$. The workstation covers are positioned to allow easy access for removal and transport of the frozen hydrated specimen grid. One press of the quick-disconnect-pushbutton on the plunge rod allows the operator to quickly and easily disconnect the plunging tweezers and safely transfer the frozen hydrated grid to a pre-cooled cryo grid storage box located within the cryo grid box transfer pot. The frozen hydrated grids are fully protected as the liquid nitrogen filled transfer pot is removed from the workstation and transferred to a liquid nitrogen storage dewar or to the pre-cooled workstation of a cryo transfer holder for low electron dose imaging on the TEM.

A typical protocol for preparing a frozen hydrated specimen using Cp3 might involve the following steps, illustrated in figure 4.

- Pre-cool the workstation and fill the ethane pot of Cp3.

- Adjust the ethane temperature so that the ethane is maintained at a temperature just above its melting point.

- Insert a pre-treated, carbon support grid (C-flat ${ }^{\mathrm{Tw}}$ and Quantifoil ${ }^{\circ}$ are commonly used) into the Cp3 plunging tweezers.

- Attach the tweezers to the plunge post and raise the plunge post to position the tweezer and grid within the humidity chamber of $\mathrm{Cp} 3$.
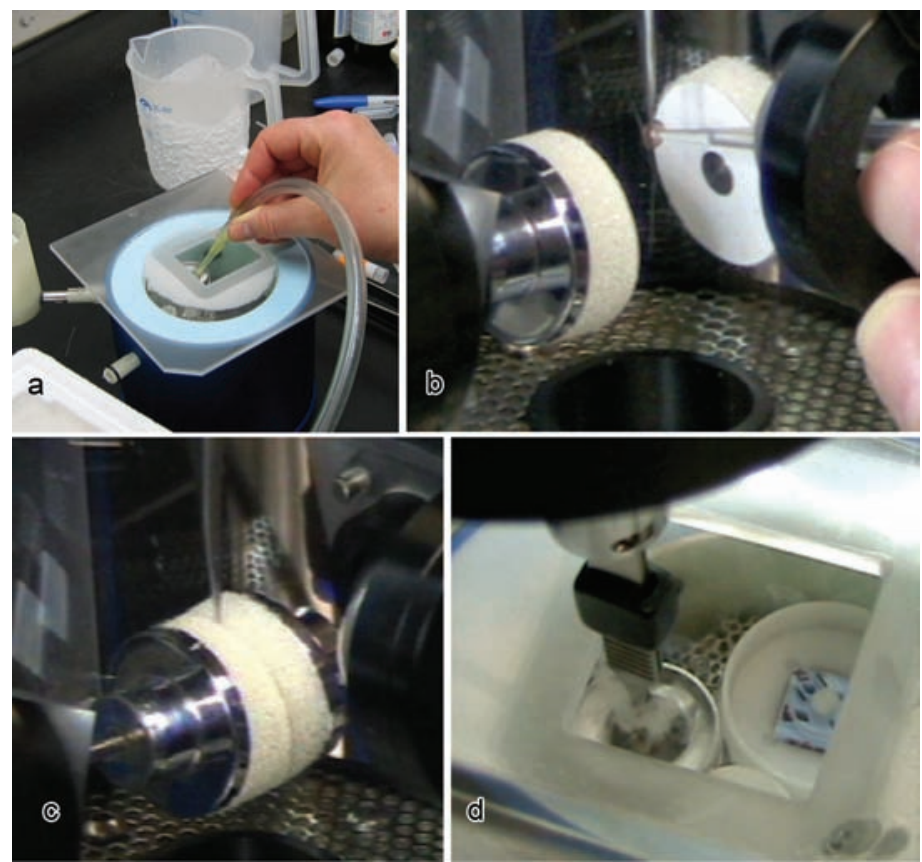

Figure 4: Condensing ethane into the pre-cooled ethane pot within the liquid nitrogen workstation (a), applying the $3 \mu \mathrm{L}$ sample aliquot to the surface of a pre-treated support film (b), 2-sided blotting of the specimen grid to produce the thin film (c), plunging the blotted grid into the ethane to freeze the sample $(d)$. 

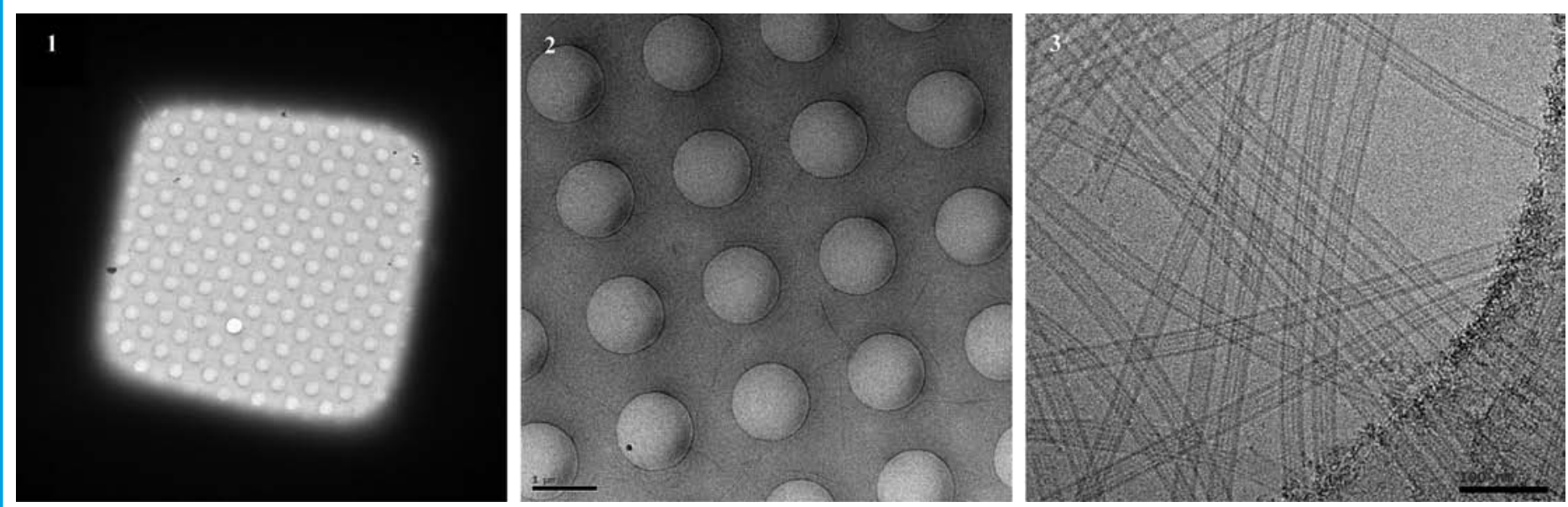

Figure 5: The three images above are an example of the high quality of frozen hydrated preparations produced with Cryoplunge ${ }^{\mathrm{TM}} 3$. (1) Image of a single grid square; TEM magnification $~ 900 X$, electron dose $0.01 \mathrm{e}^{-} / \hat{A}^{2}$. (2) Higher magnification image of a portion of the grid square; $4700 X$, $0.1 \mathrm{e}^{-} / \AA^{2}$. (3) Image of part of one hole; $59 \mathrm{KX}, 20 \mathrm{e}^{-} / \AA^{2}$. The frozen-hydrated microtubule specimen was prepared on Quantifoil ${ }^{\circledR} \mathrm{R} 1.2 / 1.3$ macro machined holey carbon grids which were plasma cleaned using the Gatan Solarus ${ }^{\circledR} 950$ Advanced Plasma Cleaning System for 15 seconds at 50 Watts using hydrogen and oxygen plasma. All images were recorded on an FEI Tecnai F30 TEM with a Gatan $62670^{\circ}$ single tilt liquid nitrogen cryo transfer holder and a Gatan Ultrascan ${ }^{\circledR} 4000$. Images courtesy of Dr. Chen Xu, Rosenstiel Basic Medical Sciences Research Center, Brandeis University, Waltham, MA.

- Insert the blotters pre-loaded with the filter paper of choice that will be used for blotting the specimen grid.

- Apply $3 \mu \mathrm{L}$ of the fluid suspension that contains the specimen to the surface of the grid.

- Set the blot timer for the desired blotting time.

- Press the start button on the Cp3; the grid is blotted to produce a thin fluid film and immediately plunged into the liquid ethane where the specimen is frozen.

- Transfer the frozen hydrated grid to a cryo grid box for storage.

- From this point forward the frozen hydrated specimen must be maintained near liquid nitrogen temperature either by storage in a liquid nitrogen dewar or by transferring it into a purpose-built cryo TEM using a cryo transfer holder maintained at liquid nitrogen temperature.

- Images of the specimen are recorded under low electron dose conditions (typically 10 to $25 \mathrm{e}^{-} / \AA^{2}$ ).

The Cryoplunge 3 is easy to use and provides clean, consistent results for the preparation of frozen hydrated specimens for cryoEM, figure 5. The $\mathrm{Cp} 3$ incorporates many of the features found in more expensive plunge freezing instruments at a fraction of the cost while providing a versatile platform to facilitate a variety of specimen preparation protocols.

\section{Acknowledgements}

The author thanks Dr. Chen Xu of Brandeis University for outstanding technical assistance and helpful discussions.

$\dagger$ Short quotes from Wikipedia contributors. Cryofixation [Internet]. Wikipedia, The Free Encyclopedia; 2008 Sep 12, 11:54 UTC [cited $2009 \mathrm{Feb}$ 1]. Available from: http://en.wikipedia. org/w/index.php?title $=$ Cryofixationsoldid $=237916959$. appear in the introductory paragraphs.

\section{General References}

Allison DP, Daw CS and Rorvik MC (1987) The construction and operation of a simple and inexpensive slam freezing device for electron microscopy. Journal of Microscopy, 147, 103-108
Bald WB (1984) The relative efficiency of cryogenic fluids used in the rapid quench-cooling of cryogenic samples. Journal of Microscopy, 134, 261-270

Bald WB (1987) Quantitative Cryofixation. Adam Hilger, Bristol and Philadelphia

Battersby BJ, Sharp JCW, Webb RI, Barnes GT (1994) Vitrification of aqueous suspensions from a controlled environment for electron microsocopy: an improved plunge-cooling device. Journal of Microscopy, 176, 110-120

Dubochet, J., Groom, M. and Mueller-Neuteboom, S. (1982), Mounting of macromolecules for electron microscopy with particular reference to surface phenomena and treatment of support films by glow discharge. Advances in optical and electron microscopy, Barrer, R. and Cosslett, V. E. (eds.), Academic Press, London, New York. 107-135.

Dubochet J, Adrian M, Chang J-J, Homo J-C, Lepault J, McDowall AW, Schulz P (1988) Cryo-electron microscopy of vitrified specimens, Quarterly Review of Biophysics 21, 129-228

Echlin P (1992) Low Temperature Microscopy and Analysis. Plenum Publishing Corporation, New York

Fukami A, Adachi K. (1965) A new method of preparation of a self-perforated micro plastic grid and its application. J Electron Microscopy (Japan). 14(2):112-118.

Glaser, R, Downing, K, DeRosier, D, Chiu, W, Fran, J. (2007) Electron Crystallography of Biological Macromolecules. Oxford University Press. 150-166.

Melanson, L. "Cryoplunge ${ }^{\mathrm{mm}} 3$ and Solarus 950: a perfect duet for consistent, high quality frozen hydrated specimen preparations for cryo transmission electron microscopy (cryoEM)." [Online]. Available: http://www.gatan.com/resources/Answers-10. php. (Nov 7, 2008).

Steinbrecht, RA, Zierold, K. (1987) Cryotechniques in biological electron microscopy. Berlin: Springer-Verlag. 47-54. 